\title{
How Much Can We Spare with E-business: Exam ining the Effects in Supply Chain Management
}

\author{
Liljana Ferbar \\ University of Ljubljana, Ljubljana, Slovenia
}

liljana.ferbar@ef.uni-lj.si

\begin{abstract}
In this paper we concentrate on one aspect of e-business applications, namely on its effects in supply chain management. We derive a mathematical model that shows how different changes in ordering costs as a result of using e-business can affect the optimal ordering intervals and quantity, average stock level and consequently total inventory-related costs. We study the sensitivity of the objective function of the average cost approach to finite magnitude perturbations of some of the system parameters which influence the optimal ordering policy. We deal with the detailed coordination case of two-level model and determine the conditions in which the model is sensitive to the variation of system parameters.
\end{abstract}

Keywords: Internet, e-business, sensitivity analysis, finite magnitude perturbations, optimal ordering policy, average cost approach

\section{Introduction}

Various aspects of e-business have attracted increased attention in recent years. Different theoretical advantages of the use of e-business were established and their practical application was described with various case studies. The question today is no longer whether to use e-business, particularly not on the business-to-business level (B2B), but how to utilize it to achieve optimal results.

In this paper we concentrate on one aspect of e-business applications, namely on its effect in supply chain management, more specifically on the possible decrease in inventory levels and total average costs. One of the main reasons for higher levels of inventory is uncertainty about the future - usually only limited information is available about the needs of suppliers, our own production and future customer wishes. E-business and specifically Internet offer an ideal way to reduce the costs of acquiring timely and exact information about those needs and possibilities. As shown by some practical examples it can indeed lead to considerably lower inventory related costs.

While the use of e-business for inventory management has attracted increased attention of researchers and practicians in recent years, we approach this topic from a slightly different angle.

We do not concentrate on methods of

Material published as part of this journal, either on-line or in print, is copyrighted by Informing Science. Permission to make digital or paper copy of part or all of these works for personal or classroom use is granted without fee provided that the copies are not made or distributed for profit or commercial advantage AND that copies 1) bear this notice in full and 2) give the full citation on the first page. It is permissible to abstract these works so long as credit is given. To copy in all other cases or to republish or to post on a server or to redistribute to lists requires specific permission from the publisher at Publisher@InformingScience.org achieving certain changes and improvements, but rather we provide a new mathematical model to show how different changes in order costs can affect optimal ordering intervals and quantity, average stock levels and consequently total inventory-related costs. 
The results, also demonstrated using a practical, numerical example in the last part of this paper, certainly support the claim that efforts in integrating e-business in supply-chain dynamics are well justified and bring substantial benefits and cost savings.

In the first part of this paper important considerations about e-business and inventory levels are briefly summarized. In the second part we briefly present the average cost approach used to determine the optimal ordering policy. In the third part of the paper we assume that order costs become perturbed and we study the effect of this finite magnitude perturbation on the optimal ordering policy. In the fourth part we demonstrate the main sensitivity results using a simple numerical example. In the last part some conclusions are given.

\section{Impact of E-business on Inventory}

In recent years more and more companies have become aware of the costs connected with inventory levels, either raw materials or finished products. In addition to the fact that a considerable amount of capital, which otherwise could be used more productively elsewhere, has to be invested in stocks, high inventory levels also cause other costs such as warehousing costs, different taxes, insurance, costs of bad quality (due to mistakes found out later), disintegration etc.

The decrease in inventory levels is possible only if the reasons for inventories are eliminated or reduced. Without this such a decrease could cause production problems, fines due to nonfulfillment of contracts and last but not least loss of customers. E-business offers multiple possibilities for the decrease in inventory levels (Van der Vorst, Van Dongen, Nouguier, \& Hilhorst, 2002). Specifically it can help to reduce order costs, which leads to the decrease in total inventory costs, as shown in this paper.

The main advantage of Internet and e-business is that it can make information widely available at low costs. This means that inventory levels can be reduced at all levels of supply chain without increasing the risk of stockouts (and costs related with stock-outs) for any company or customer involved in the process.

IT enables new ways of manufacturing, such as just-in-time production or virtual enterprise system (more about virtual enterprises can be found for example in Krauter (1999)) that can result in a considerable decrease in inventory and inventory related costs. The precondition for that is good cooperation of all links in the supply chain - the company has to assure quick, cost-effective and reliable system of communication with its customers and suppliers (Zupancic, 1997). Needless to say, Internet offers an ideal way to do this.

Additionaly Internet can significantly lower transaction costs and consequently, due to lower costs per order, also the optimal ordering quantitiy and average inventory levels (Trombly, 2000). According to a basic formula:

$$
\text { optimal ordering quantity }=\sqrt{\frac{2 * \text { yearly demand } * \text { order costs }}{\text { yearly cost of one unit in stock }}}
$$

a decrease in order costs by $50 \%$ decreases the optimal order size, and consequently inventory levels, by $30 \%$. In addition to e-business, production automation can also lower other costs.

For additional decrease of inventory levels it is also crucial to lower the time between order placement and material delivery. Information technology enables different new ordering systems such as vendor-managed inventory, where the supplier is in charge of monitoring the inventory level and delivering the required material on time. Paper-based documentation is therefore not needed anymore and the supply time can be reduced - according to some estimation even from 
21-29 days to 14-17 days (Emigh, 1999). Consequently, security levels of stocks can also be reduced, without risking possible troubles in production due to lack of needed material.

Different studies have shown that electronic data interchange and e-business can indeed lead to a substantial inventory levels decrease (see McCubrrey (1998) and Goutsos, \& Karacapilidis (2003)). The studies indicate that inventory levels of companies that are leading their supply chain dynamics are, on average, 50-80\% lower than those of other companies in the same branch.

However, an extensive study of different Slovenian companies found no significant correlations between investment in information technology and the use of e-business on the one side, and inventory levels or inventory changes on the other (Trkman, 2000). As noted in Trkman, Turk, Vehovar, \& Jerman-Blazic (2001), this should not lead to a conclusion that e-business is not a proper way to reduce inventory-related costs, but rather to the fact that Slovenian firms are not using this possibility to decrease inventory properly. Ideally the use of information technology and ebusiness should be accompanied by careful business process engineering and obviously full support from the management.

As there are plenty of different case studies available (in addition to already mentioned also see Panayiotou, Gaxialis, \& Tatsiopoulos (2003) and Ramani, Yap, \& Pavri (1995)) we will not concentrate on specific case study or specific way to obtain competitive advantage due to the decrease in inventory level as a consequence of IT or e-business. Rather we will develop a new mathematical model to show how different changes achieved with the proper use of e-business can lead to a decrease in inventory levels and consequently decrease in associated costs.

\section{Average Cost Approach}

From time to time we encounter different sensitivity studies in available literature on MRP (Material Requirement Planning), as in Sianesi \& Zavanella (1996) for example. While in their study a simulative investigation on sensitivity of system parameters is given, our approach is quite different. For the system parameter variations of finite magnitude we explicitly calculate the corresponding absolute and relative change in the value of the objective function (the change in the value of total average costs). The results are particularly important for quick investigation of the influence of finite magnitude change to the value of the objective function (Ferbar \& Bogataj, 1997).

Let us introduce the following notation:

$N=$ number of different items including raw materials, components, $\ldots$

$Q_{i}=$ order quantity of item $i, i=1,2, \ldots, N$

$T_{i}=$ ordering interval (cycle time) of item $i, i=1,2, \ldots, N$

$\kappa_{i}=$ number of batch completions of item $i$ at some point in time

$d_{i}=$ external demand rate of item $i$ at some time

$S_{i}=$ total inventory of item $i$ at some time

$H_{i j}=$ number of units of item $i$ required for the production of one unit of item $j$

$\varepsilon_{i}=$ the earliest time at which the demand $d_{i}$ can be met

$\Delta_{i}=$ time at which the first completion of item $i$ occurs

$\mu_{i}=$ unit batch completion of item $i$ beginning at time zero

$K_{i}=$ the setup cost of item $i$

$v_{i}=$ inventory value of item $i$

$\rho=$ continuous interest rate 
For convenience, we arrange the above quantities into vectors and matrices according to $Q, T, \delta$, $\Delta$ being $(N \mathrm{x} N)$-dimensional diagonal matrices with $Q_{i}, T_{i}, \delta_{i}$ and $\Delta_{i}$ in $i$ th diagonal position respectively; $H$ an $(N \mathrm{x} N)$-dimensional matrix with element $H_{i j}$ in position $(i, j) ; H^{*}=(I-H)^{-1}$ being the Leontief inverse of $H ; \kappa, \mu, d$ and $S$ all being $N$-dimensional column vectors with $i$ th components $\kappa_{i}, \mu_{i}, d_{i}$ and $S_{i}$ respectively; $K$ and $v$ being $N$-dimensional row vectors with $i$ th components $K_{i}$ and $v_{i}$ respectively.

Following the traditional approach (Grubbstrom \& Molinder, 1994) the total average cost can be written as the sum of total average order costs and total average holding costs:

$$
C_{0}=K \bar{\mu}+\rho v \bar{S},
$$

where $\bar{\mu}_{i}=\frac{1}{T_{i}}$ and $\bar{S}=S(0)-(I-H)(\Delta-T / 2) H^{*} d+\delta d$.

The first term decreases inversely with $T_{i}$ and the other increases linearly. Differentiating with respect to an individual $T_{i}$ :

$$
\frac{\partial C_{0}}{\partial T_{i}}=-\frac{K_{i}}{T_{i}^{2}}+\frac{\rho v_{i}}{2}(I-H)_{i} H_{i}^{*} d=0
$$

gives us the optimal free ordering intervals $\left(T_{0}^{*}\right)_{i}$ and economic order quantities $\left(Q_{0}^{*}\right)_{i}$ :

$$
\left(T_{0}^{*}\right)_{i}=\sqrt{\frac{2 K_{i}}{\rho v_{i}(I-H)_{i} H_{i}^{*} d}} \text { and }\left(Q_{0}^{*}\right)_{i}=\left(T_{0}^{*}\right)_{i} H_{i}^{*} d=\sqrt{\frac{2 K_{i} H_{i}^{*} d}{\rho v_{i}(I-H)_{i}}} .
$$

In this case the optimal total costs are:

$$
C_{0}^{*}=\sum_{i=1}^{N}\left[\sqrt{2 K_{i} \rho v_{i}(I-H)_{i} H_{i}^{*} d}+\rho v_{i}\left(S_{i}(0)-\Delta_{i}(I-H)_{i} H_{i}^{*} d+\delta_{i} d_{i}\right)\right] .
$$

Let us assume that order costs $K$ become perturbed (reduced) with the use of e-business (either between companies and/or within production), so that their new value is $K-\Delta K$ (and $\Delta K$ being the magnitude of the perturbation: $\left.\Delta K=\left(\Delta K_{1}, \ldots, \Delta K_{N}\right)\right)$. In this case the total average perturbed costs can be written as:

$$
C_{p}=(K-\Delta K) \bar{\mu}+\rho v \bar{S} .
$$

Differentiating with respect to $T_{i}$ get us:

$$
\frac{\partial C_{p}}{\partial T_{i}}=-\frac{K_{i}-\Delta K_{i}}{T_{i}^{2}}+\frac{\rho v_{i}}{2}(I-H)_{i} H_{i}^{*} d=0
$$

and from this we can calculate the perturbed optimal free ordering intervals $\left(T_{p}^{*}\right)_{i}$ and the perturbed optimal economic order quantities $\left(Q_{p}^{*}\right)_{i}$ :

$$
\left(T_{p}^{*}\right)_{i}=\sqrt{\frac{2\left(K_{i}-\Delta K_{i}\right)}{\rho v_{i}(I-H)_{i} H_{i}^{*} d}} \text { and }\left(Q_{p}^{*}\right)_{i}=\left(T_{p}^{*}\right)_{i} H_{i}^{*} d=\sqrt{\frac{2\left(K_{i}-\Delta K_{i}\right) H_{i}^{*} d}{\rho v_{i}(I-H)_{i}}}
$$


In this case the optimal perturbed total costs are:

$$
C_{p}^{*}=\sum_{i=1}^{N}\left[\sqrt{2\left(K_{i}-\Delta K_{i}\right) \rho v_{i}(I-H)_{i} H_{i}^{*} d}+\rho v_{i}\left(S_{i}(0)-\Delta_{i}(I-H)_{i} H_{i}^{*} d+\delta_{i} d_{i}\right)\right]
$$

Now we calculate the perturbation of the optimal free ordering intervals:

$$
\Delta T_{i}^{*}=\left(T_{0}^{*}\right)_{i}-\left(T_{p}^{*}\right)_{i}=\left(T_{0}^{*}\right)_{i} \frac{\frac{\Delta K_{i}}{K_{i}}}{1+\sqrt{1-\frac{\Delta K_{i}}{K_{i}}}}
$$

and its relative change:

$$
\frac{\Delta T_{i}^{*}}{\left(T_{0}^{*}\right)_{i}}=\frac{\left(T_{0}^{*}\right)_{i}-\left(T_{p}^{*}\right)_{i}}{\left(T_{0}^{*}\right)_{i}}=\frac{\frac{\Delta K_{i}}{K_{i}}}{1+\sqrt{1-\frac{\Delta K_{i}}{K_{i}}}}
$$

In a similar way we get the perturbation and the relative change of the optimal economic order quantities:

$$
\Delta Q_{i}^{*}=\left(Q_{0}^{*}\right)_{i}-\left(Q_{p}^{*}\right)_{i}=\left(Q_{0}^{*}\right)_{i} \frac{\frac{\Delta K_{i}}{K_{i}}}{1+\sqrt{1-\frac{\Delta K_{i}}{K_{i}}}} \text { and } \frac{\Delta Q_{i}^{*}}{\left(Q_{0}^{*}\right)_{i}}=\frac{\frac{\Delta K_{i}}{K_{i}}}{1+\sqrt{1-\frac{\Delta K_{i}}{K_{i}}}}
$$

Finally we calculate the perturbation of the optimal total average costs as:

$$
\begin{aligned}
\Delta C^{*} & =C_{0}^{*}-C_{p}^{*}=\sum_{i=1}^{N}\left[\sqrt{2 \rho v_{i}(I-H)_{i} H_{i}^{*} d} \cdot \frac{\Delta K_{i}}{\sqrt{K_{i}}+\sqrt{K_{i}-\Delta K_{i}}}\right]= \\
& =\sum_{i=1}^{N}\left[\sqrt{2 K_{i} \rho v_{i}(I-H)_{i} H_{i}^{*} d} \cdot \frac{\frac{\Delta K_{i}}{K_{i}}}{1+\sqrt{1-\frac{\Delta K_{i}}{K_{i}}}}\right]
\end{aligned}
$$

and its relative change:

$$
\frac{\Delta C^{*}}{C_{0}^{*}}=\frac{\sum_{i=1}^{N}\left[\frac{\frac{\Delta K_{i}}{K_{i}}}{1+\sqrt{1-\frac{\Delta K_{i}}{K_{i}}}} \sqrt{2 K_{i} \rho v_{i}(I-H)_{i} H_{i}^{*} d}\right]}{\sum_{i=1}^{N}\left[\sqrt{2 K_{i} \rho v_{i}(I-H)_{i} H_{i}^{*} d}+\rho v_{i}\left(S_{i}(0)-\Delta_{i}(I-H)_{i} H_{i}^{*} d+\delta_{i} d_{i}\right)\right]} .
$$

\section{The Detailed Coordination Case of Two-Level Model}

Let us consider the detailed coordination case of the two-level model (so called GrubbströmMolinder model) with average cost approach (for details see also Grubbstrom \& Molinder (1994), Bogataj \& Ferbar (1996)). We have in this case:

$$
H=\left[\begin{array}{ll}
0 & 0 \\
1 & 0
\end{array}\right], \quad I-H=\left[\begin{array}{cc}
1 & 0 \\
-1 & 1
\end{array}\right], \quad H^{*}=(I-H)^{-1}=\left[\begin{array}{ll}
1 & 0 \\
1 & 1
\end{array}\right] \text { and } H^{*} d=\left[\begin{array}{ll}
1 & 0 \\
1 & 1
\end{array}\right]\left[\begin{array}{c}
d_{1} \\
0
\end{array}\right]=\left[\begin{array}{l}
d_{1} \\
d_{1}
\end{array}\right] \text {. }
$$


We presume that we have one final product which contains only one component. Lead-times are equal to zero, initial inventory $S(0)=0$, external demand for final product is $d_{1}$ and there is no external demand for the component.

As we know from previous findings (Grubbstrom \& Molinder, 1994; Bogataj \& Ferbar, 1996; Ferbar \& Bogataj, 1999) the total costs can be minimized when the condition $T_{2}=m T_{1}$, where $m$ is an integer, is fulfilled. Also, we can choose the model so that the shortest horizontal starting distance is $\hat{\Delta}^{*}=0\left(\hat{\Delta}=\Delta_{1}-\Delta_{2}\right)$. In this case the total costs are:

$$
\begin{aligned}
C_{0}\left(T_{1}, m\right) & =K \bar{\mu}+\rho v \bar{S}=\left[\begin{array}{ll}
K_{1} & K_{2}
\end{array}\right]\left[\begin{array}{c}
\frac{1}{T_{1}} \\
\frac{1}{T_{2}}
\end{array}\right]+\rho\left[\begin{array}{ll}
v_{1} & v_{2}
\end{array}\right]\left[\begin{array}{cc}
1 & 0 \\
-1 & 1
\end{array}\right]\left[\begin{array}{cc}
\frac{T_{1}}{2} & 0 \\
0 & \frac{T_{2}}{2}
\end{array}\right]\left[\begin{array}{cc}
1 & 0 \\
1 & 1
\end{array}\right]\left[\begin{array}{c}
d_{1} \\
0
\end{array}\right]= \\
& =\frac{K_{1}}{T_{1}}+\frac{K_{2}}{m T_{1}}+\rho v_{1} \frac{d_{1} T_{1}}{2}+\rho v_{2} \frac{d_{1} T_{1} m}{2}-\rho v_{2} \frac{d_{1} T_{1}}{2}
\end{aligned}
$$

To achieve the optimal value, the following necessary conditions of optimality must be fulfilled: $\frac{\partial C_{0}}{\partial T_{1}}=0$ and $\frac{\partial C_{0}}{\partial m}=0$. So, we get:

$$
\left(T_{0}^{*}\right)_{1}=\sqrt{\frac{2\left(K_{1}+\frac{K_{2}}{m_{0}^{*}}\right)}{\rho d_{1}\left(v_{1}+v_{2}\left(m_{0}^{*}-1\right)\right)}} \text { and } m_{0}^{*}=\sqrt{\frac{K_{2}\left(v_{1}-v_{2}\right)}{K_{1} v_{2}}} .
$$

Let us note that for $m_{0}^{*}$ in practice the number rounded up is taken (Grubbstrom-Molinder, 1994). The optimal total average costs have the following form:

$$
C_{0}^{*}=\sqrt{2 \rho d_{1}\left(K_{1}+\frac{K_{2}}{m_{0}^{*}}\right)\left(v_{1}+v_{2}\left(m_{0}^{*}-1\right)\right)} .
$$

\section{The Main Sensitivity Results for Finite Magnitude Perturbation}

Considering that the total change of order costs is $\Delta K$ (we suppose that value of order costs can be reduced with the use of e-business, either between companies and/or within production), the total average perturbed costs can be expressed as:

$$
C_{p}\left(T_{1}, m\right)=\frac{1}{T_{1}}\left(K_{1}-\Delta K_{1}+\frac{K_{2}-\Delta K_{2}}{m_{p}}\right)+\frac{\rho d_{1} T_{1}}{2}\left(v_{1}+v_{2}\left(m_{p}-1\right)\right) .
$$

From the necessary conditions of optimality: $\frac{\partial C_{p}}{\partial T_{1}}=0$ and $\frac{\partial C_{p}}{\partial m_{p}}=0$, we get:

$$
\left(T_{p}^{*}\right)_{1}=\sqrt{\frac{2\left(K_{1}-\Delta K_{1}+\frac{K_{2}-\Delta K_{2}}{m_{p}^{*}}\right)}{\rho d_{1}\left(v_{1}+v_{2}\left(m_{p}^{*}-1\right)\right)}} \text { and } m_{p}^{*}=\sqrt{\frac{\left(K_{2}-\Delta K_{2}\right)\left(v_{1}-v_{2}\right)}{\left(K_{1}-\Delta K_{1}\right) v_{2}}} \text {. }
$$


Using these optimal perturbed values in $C_{p}\left(T_{1}, m\right)$ we get the following optimal value of total average perturbed costs:

$$
C_{p}^{*}=\sqrt{2 \rho d_{1}\left(K_{1}-\Delta K_{1}+\frac{K_{2}-\Delta K_{2}}{m_{p}^{*}}\right)\left(v_{1}+v_{2}\left(m_{p}^{*}-1\right)\right)},
$$

so the absolute change in costs is:

$$
\Delta C=C_{0}^{*}-C_{p}^{*}=\frac{\sqrt{2 \rho d_{1}}\left[\left(\Delta K_{1}+\frac{\Delta K_{2}}{m_{p}^{*}}\right)\left(v_{1}+v_{2}\left(m_{p}^{*}-1\right)\right)+\left(m_{0}^{*}-m_{p}^{*}\right)\left(K_{1} v_{2}+\frac{K_{2}\left(v_{2}-v_{1}\right)}{m_{0}^{*} m_{p}^{*}}\right)\right]}{\sqrt{\left(K_{1}+\frac{K_{2}}{m_{0}^{*}}\right)\left(v_{1}+v_{2}\left(m_{0}^{*}-1\right)\right)}+\sqrt{\left(K_{1}-\Delta K_{1}+\frac{K_{2}-\Delta K_{2}}{m_{p}^{*}}\right)\left(v_{1}+v_{2}\left(m_{p}^{*}-1\right)\right)}}
$$

Using the following relation between $m_{p}^{*}$ and $m_{0}^{*}$ :

$$
m_{p}^{*}=\sqrt{\frac{\left(K_{2}-\Delta K_{2}\right)\left(v_{1}-v_{2}\right)}{\left(K_{1}-\Delta K_{1}\right) v_{2}}}=\sqrt{\frac{K_{2}\left(v_{1}-v_{2}\right)}{K_{1} v_{2}}} \sqrt{\frac{K_{1}\left(K_{2}-\Delta K_{2}\right)}{K_{2}\left(K_{1}-\Delta K_{1}\right)}}=m_{0}^{*} \sqrt{\frac{1-\frac{\Delta K_{2}}{K_{2}}}{1-\frac{\Delta K_{1}}{K_{1}}}},
$$

we can derive the relative change in $m_{0}^{*}$ as:

$$
\frac{\Delta m^{*}}{m_{0}^{*}}=\frac{\left|m_{0}^{*}-m_{p}^{*}\right|}{m_{0}^{*}}=\left|1-\sqrt{\frac{1-\frac{\Delta K_{2}}{K_{2}}}{1-\frac{\Delta K_{1}}{K_{1}}}}\right| .
$$

From Figure 1 we can see that $m_{p}^{*}$ can be reduced in comparison with $m_{0}^{*}$ by $75 \%$ at most and

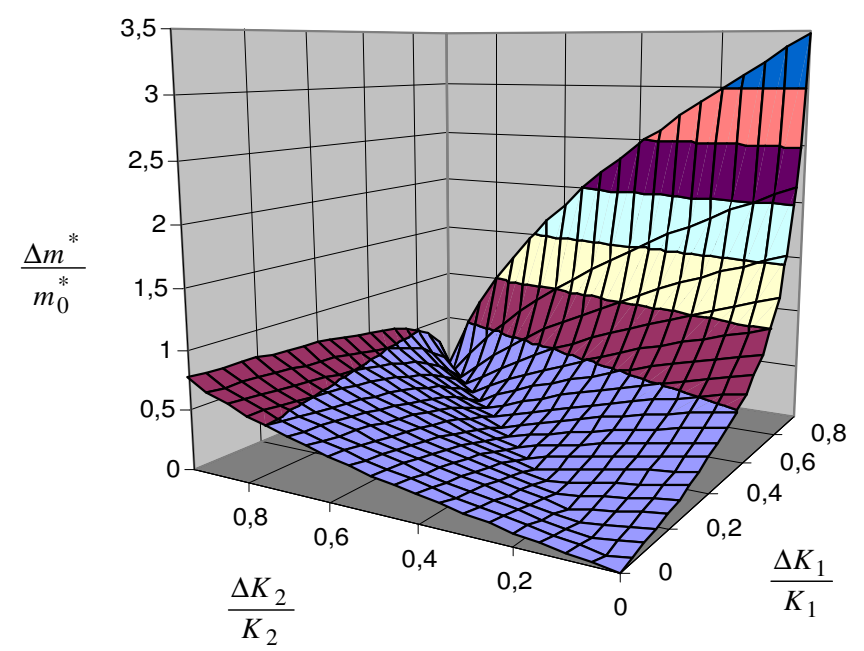

Figure 1: The diagram of relative changes of parameter $\boldsymbol{m}\left(\frac{\Delta m^{*}}{m_{0}^{*}}\right)$ as a function of $\frac{\Delta K_{1}}{K_{1}}$ and $\frac{\Delta K_{2}}{K_{2}}$. 
can be increased by almost $375 \%$ (this means that parameter $m_{p}^{*}$ could be nearly four times grater than $m_{0}^{*}$ for some perturbations of order costs). In Figure 2 we present the relative changes of parameter $m$ for two special cases when $\frac{\Delta K_{2}}{K_{2}}=0.5$ and when $\frac{\Delta K_{1}}{K_{1}}=0.5$.
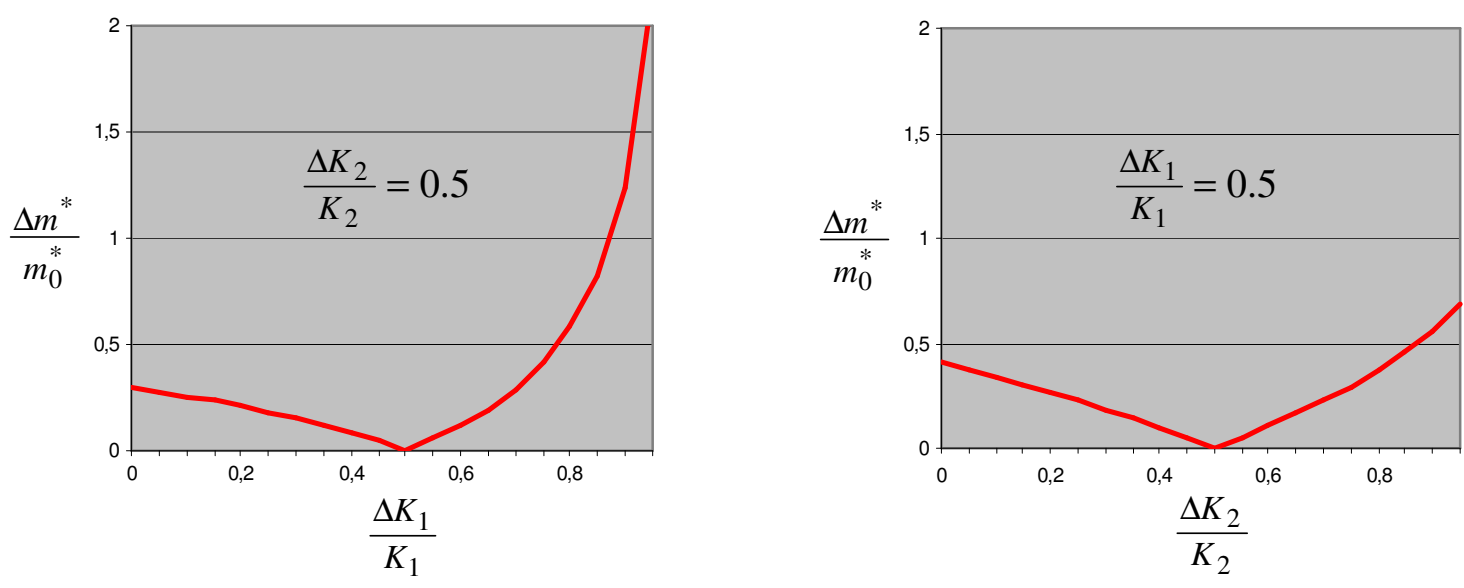

Figure 2: The diagram of relative changes of parameter $\boldsymbol{m}\left(\frac{\Delta m^{*}}{m_{0}^{*}}\right)$ as

a function of $\frac{\Delta K_{1}}{K_{1}}$ and $\frac{\Delta K_{2}}{K_{2}}$ for the cases when $\frac{\Delta K_{2}}{K_{2}}=0.5$ and when $\frac{\Delta K_{1}}{K_{1}}=0.5$.

We will observe only those perturbations which cause the absolute changes in $m_{p}^{*}$ for 1 at most:

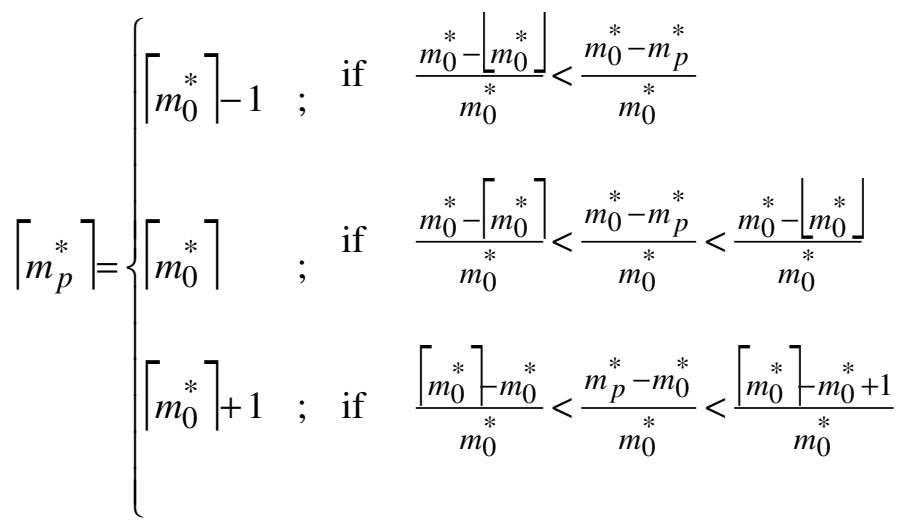

or (if we consider the relation between $m_{p}^{*}$ and $m_{0}^{*}$ ) 


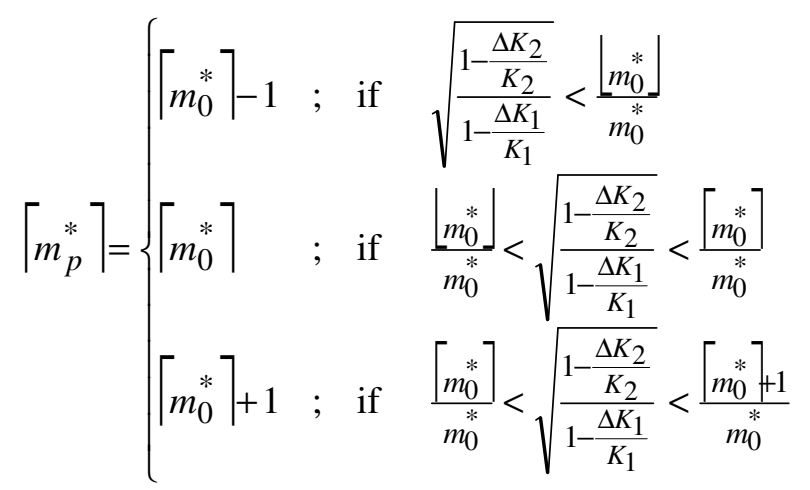

where notation $\lceil\cdot\rceil$ means the number rounded up and $\lfloor\cdot\rfloor$ the number rounded down.

\section{A Simple Numerical Example}

Let us consider the case where the model parameters are:

$\rho=0.1, d_{1}=100, v_{1}=30, v_{2}=10, K_{1}=20$ and $K_{2}=15$.

Then

$$
\begin{aligned}
& m_{0}^{*}=\sqrt{\frac{15 \cdot 20}{20 \cdot 10}}=\sqrt{1,5}=1,225 \text { and }\left[m_{0}^{*} \mid=2,\right. \\
& \left(T_{0}^{*}\right)_{1}=\sqrt{\frac{2\left(20+\frac{15}{2}\right)}{10(30+10)}}=\sqrt{\frac{55}{400}}=0,37, \\
& C_{0}^{*}=\sqrt{20\left(20+\frac{15}{2}\right)(30+10)}=20 \sqrt{55}=148,32 .
\end{aligned}
$$

First we look at the case where $\left|m_{p}^{*}\right|=\left|m_{0}^{*}\right|-1=1$, so the condition (1) is fulfilled (we suppose that relative changes of order costs, which are the result of the use of e-business, fulfil the following condition: $\frac{\Delta K_{2}}{K_{2}}>\frac{1}{3}+\frac{2}{3} \cdot \frac{\Delta K_{1}}{K 1}$; for example, if the order costs on the first level can be reduced by $50 \%$, the order costs on the second level should be reduced by at least $67 \%$ ). In this case, the optimal perturbed ordering interval is:

$$
\left(T_{p}^{*}\right)_{1}=\sqrt{\frac{2\left(20-\Delta K_{1}+\frac{15-\Delta K_{2}}{1}\right)}{10(30+10(1-1))}}=\sqrt{\frac{35-\Delta K_{1}-\Delta K_{2}}{150}}
$$

and the relative change is:

$$
\frac{\Delta T_{1}^{*}}{\left(T_{0}^{*}\right)_{1}}=\frac{\left|\left(T_{0}^{*}\right)_{1}-\left(T_{p}^{*}\right)_{1}\right|}{\left(T_{0}^{*}\right)_{1}}=\left|1-\sqrt{\frac{8\left(35-\Delta K_{1}-\Delta K_{2}\right)}{165}}\right|=\left|1-\sqrt{\frac{8\left(7-4 \cdot \frac{\Delta K_{1}}{K_{1}}-3 \cdot \frac{\Delta K_{2}}{K_{2}}\right)}{33}}\right| .
$$

We can see from equation above, that for relative changes of $\frac{\Delta K_{1}}{K_{1}}$ and $\frac{\Delta K_{2}}{K_{2}}$, which fulfil the additional condition $\frac{1}{24}\left(23-32 \frac{\Delta K_{1}}{K_{1}}\right)<\frac{\Delta K_{2}}{K_{2}}$, the value of optimal perturbed ordering interval $\left(T_{p}^{*}\right)_{1}$ will be reduced and so the optimal perturbed order quantity $\left(Q_{p}^{*}\right)_{1}$. For other perturbations of order costs the optimal perturbed ordering interval will increase, but as we will see from the 
next derivation the total perturbed costs will decrease in both cases. If we calculate the optimal total average perturbed costs:

$$
\left(C_{p}^{*}\right)_{1}=10 \sqrt{6\left(35-\Delta K_{1}-\Delta K_{2}\right)}
$$

and its relative change:

$$
\frac{\Delta C_{1}^{*}}{C_{0}^{*}}=\frac{\left|C_{0}^{*}-\left(C_{p}^{*}\right)_{1}\right|}{C_{0}^{*}}=\left|1-\sqrt{\frac{3\left(35-\Delta K_{1}-\Delta K_{2}\right)}{110}}\right|=\left|1-\sqrt{\frac{3\left(7-4 \cdot \frac{\Delta K_{1}}{K_{1}}-3 \cdot \frac{\Delta K_{2}}{K_{2}}\right)}{22}}\right|,
$$

we can conclude that the total costs can be reduced for any relative changes of order costs which fulfil the condition (1) what we can see also in Figure 3. For example, if the order costs on the first level can be reduced by $50 \%$ and the order costs on the second level by at least $67 \%$, then the total costs can be reduced by at least $36 \%$. This reduction of the total costs is the consequence of the decrease of the optimal ordering interval on the second level $\left(\left(T_{0}^{*}\right)_{2}=2 \cdot\left(T_{0}^{*}\right)_{1}\right.$ is reduced to $\left.\left(T_{p}^{*}\right)_{2}=1 \cdot\left(T_{p}^{*}\right)_{1}\right)$.
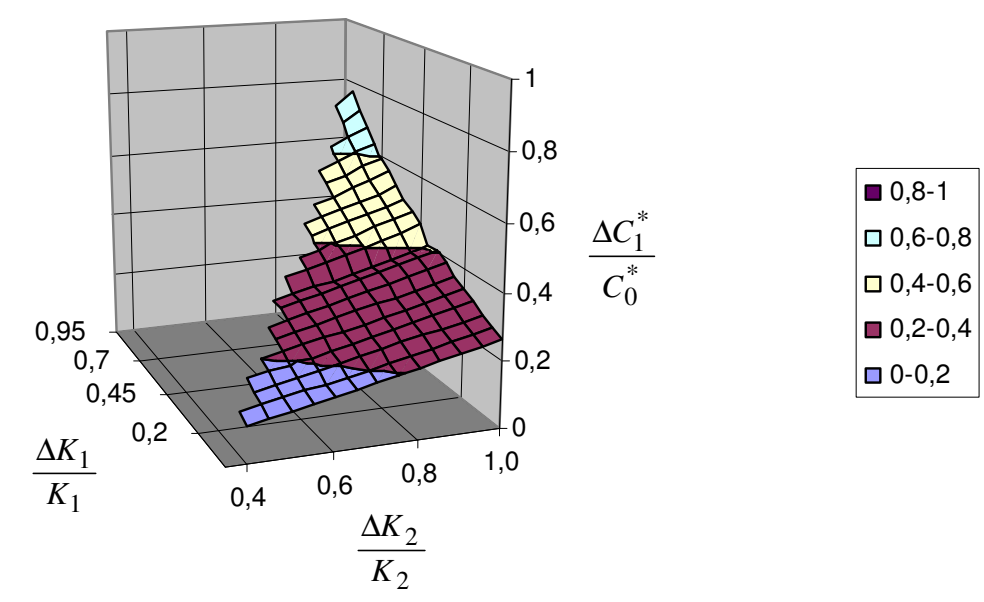

Figure 3: The diagram of the relative change of the optimal total average costs $\left(\frac{\Delta C_{1}^{*}}{C_{0}^{*}}\right)$ as a function of $\frac{\Delta K_{1}}{K_{1}}$ and $\frac{\Delta K_{2}}{K_{2}}$.

In the case when $\left|m_{p}^{*}\right|=\left|m_{0}^{*}\right|=2$ (we suppose that relative changes of order costs fulfil the condition (2): $-\frac{5}{3}+\frac{8}{3} \cdot \frac{\Delta K_{1}}{K 1}<\frac{\Delta K_{2}}{K_{2}}<\frac{1}{3}+\frac{2}{3} \cdot \frac{\Delta K_{1}}{K 1}$; for example, if the order costs on the first level can be reduced by $70 \%$, the order costs on the second level should be reduced by at least $20 \%$ but not more than $80 \%$ ) we get:

$$
\left(T_{p}^{*}\right)_{1}=\sqrt{\frac{2\left(20-\Delta K_{1}+\frac{15-\Delta K_{2}}{2}\right)}{10(30+10)}}=\sqrt{\frac{55-2 \Delta K_{1}-\Delta K_{2}}{400}}
$$

and: 


$$
\frac{\Delta T_{1}^{*}}{\left(T_{0}^{*}\right)_{1}}=\frac{\left|\left(T_{0}^{*}\right)_{1}-\left(T_{p}^{*}\right)_{1}\right|}{\left(T_{0}^{*}\right)_{1}}=\left|1-\sqrt{\frac{\left.55-2 \Delta K_{1}-\Delta K_{2}\right)}{55}}\right|=\left|1-\sqrt{\frac{11-8 \cdot \frac{\Delta K_{1}}{K_{1}}-3 \cdot \frac{\Delta K_{2}}{K_{2}}}{11}}\right| .
$$

The optimal value of the perturbed total average costs in this case is:

$$
\left(C_{p}^{*}\right)_{2}=20 \sqrt{55-2 \Delta K_{1}-\Delta K_{2}}
$$

so its relative change can be written as:

$$
\frac{\Delta C_{2}^{*}}{C_{0}^{*}}=\frac{\left|C_{0}^{*}-\left(C_{p}^{*}\right)_{2}\right|}{C_{0}^{*}}=\left|1-\sqrt{\frac{55-2 \Delta K_{1}-\Delta K_{2}}{55}}\right|=\left|1-\sqrt{\frac{11-8 \cdot \frac{\Delta K_{1}}{K_{1}}-3 \cdot \frac{\Delta K_{2}}{K_{2}}}{11}}\right| .
$$

From the equation above and from Figure 4 we can see that the optimal total perturbed costs can be reduced for any relative changes of $\frac{\Delta K_{1}}{K_{1}}$ and $\frac{\Delta K_{2}}{K_{2}}$ which fulfil the condition (2).

For example, if the order costs on the first level can be reduced by $70 \%$ and the order costs on the second level by at least $20 \%$, then the total costs can be reduced by $34 \%$ at least (if the order costs

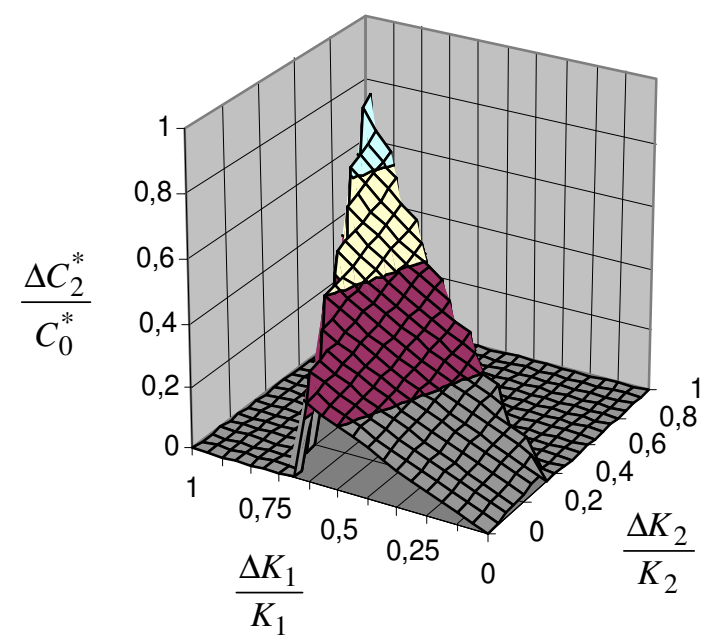

$\square 0,8-1$

$\square 0,6-0,8$

$\square 0,4-0,6$

$\square$ 0,2-0,4

$\square 0-0,2$

Figure 4: The diagram of relative change of the optimal total average costs

$$
\left(\frac{\Delta C_{2}^{*}}{C_{0}^{*}}\right) \text { as a function of } \frac{\Delta K_{1}}{K_{1}} \text { and } \frac{\Delta K_{2}}{K_{2}} \text {. }
$$

on the second level can be reduced by $80 \%$, the total costs can be reduced by almost $48 \%$ ). In this case the optimal ordering interval on the second level is still two times grater than optimal ordering interval on the first level $\left(\left(T_{p}^{*}\right)_{2}=2 \cdot\left(T_{p}^{*}\right)_{1}\right)$, but it becomes smaller together with $\left(T_{p}^{*}\right)_{1}$ because of the reduction of order costs.

When $\left|m_{p}^{*}\right|=\left|m_{0}^{*}\right|+1=3$ (we suppose that relative changes of order costs fulfil the condition (3): $\frac{\Delta K_{2}}{K_{2}}<-\frac{5}{3}+\frac{8}{3} \cdot \frac{\Delta K_{1}}{K 1}$; for example, if the order costs on the first level can be reduced by $70 \%$, the 
order costs on the second level should be reduced less than 20\%), the optimal perturbed ordering interval is:

$$
\left(T_{p}^{*}\right)_{1}=\sqrt{\frac{2\left(20-\Delta K_{1}+\frac{15-\Delta K_{2}}{3}\right)}{10(30+10(3-1))}}=\sqrt{\frac{75-3 \Delta K_{1}-\Delta K_{2}}{750}}
$$

and the relative change is:

$$
\frac{\Delta T_{1}^{*}}{\left(T_{0}^{*}\right)_{1}}=\frac{\left|\left(T_{0}^{*}\right)_{1}-\left(T_{p}^{*}\right)_{1}\right|}{\left(T_{0}^{*}\right)_{1}}=\left|1-\sqrt{\frac{8\left(75-3 \Delta K_{1}-\Delta K_{2}\right)}{825}}\right|=\left|1-\sqrt{\frac{8\left(5-4 \cdot \frac{\Delta K_{1}}{K_{1}}-\frac{\Delta K_{2}}{K_{2}}\right)}{55}}\right| .
$$

From this expression we can see that the optimal ordering interval $\left(T_{p}^{*}\right)_{1}$ can be reduced in comparison with $\left(T_{0}^{*}\right)_{1}$ by $15 \%$ at least. In this case the optimal ordering interval on the second level can increase by $27,5 \%$ and it can cause the increase of total average costs. The interval $\left(T_{p}^{*}\right)_{2}$ would stay unchanged in comparison with $\left(T_{0}^{*}\right)_{2}$, if $\left(T_{p}^{*}\right)_{1}$ would decrease with regard to $\left(T_{0}^{*}\right)_{1}$ by $33,3 \%$ at least, what we can derive from $\left(T_{0}^{*}\right)_{2}=2\left(T_{0}^{*}\right)_{1}=3\left(1-\frac{x}{100}\right)\left(T_{0}^{*}\right)_{1}$.

For this third case, when $\left|m_{p}^{*}\right|=3$, the optimal total average perturbed costs are:

$$
\left(C_{p}^{*}\right)_{3}=10 \sqrt{\frac{10}{3}\left(75-3 \Delta K_{1}-\Delta K_{2}\right)}
$$

and its relative change is:

$$
\frac{\Delta C_{3}^{*}}{C_{0}^{*}}=\frac{\left|C_{0}^{*}-\left(C_{p}^{*}\right)_{3}\right|}{C_{0}^{*}}=\left|1-\sqrt{\frac{5\left(75-3 \Delta K_{1}-\Delta K_{2}\right.}{330}}\right|=\left|1-\sqrt{\frac{5\left(5-4 \cdot \frac{\Delta K_{1}}{K_{1}}-\frac{\Delta K_{2}}{K_{2}}\right)}{22}}\right|
$$

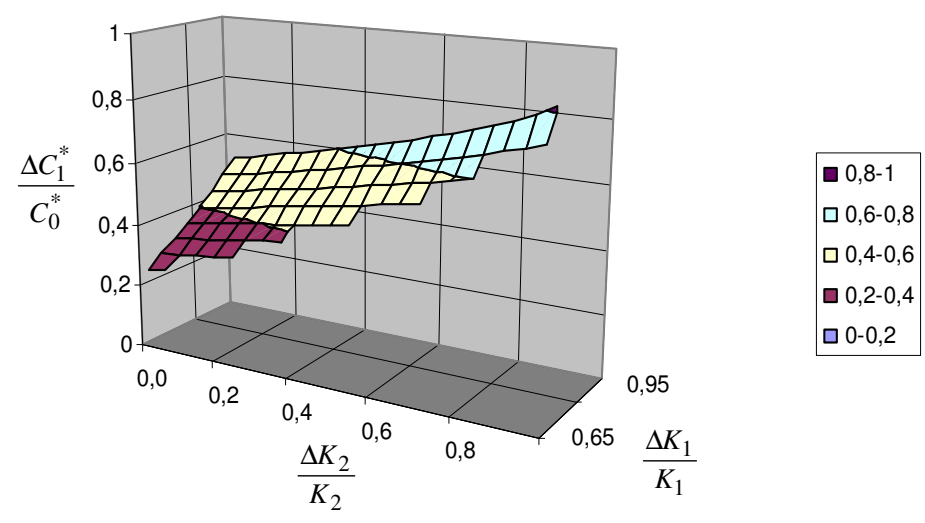

Figure 5: The diagram of the relative change of the optimal total average $\operatorname{costs}\left(\frac{\Delta C_{3}^{*}}{C_{0}^{*}}\right)$ as a function of $\frac{\Delta K_{1}}{K_{1}}$ and $\frac{\Delta K_{2}}{K_{2}}$. 
As we can see from equation (8), the optimal total perturbed costs can be reduced if the additional condition $0,6-4 \cdot \frac{\Delta K_{1}}{K_{1}}<\frac{\Delta K_{2}}{K_{2}}$ is valid. But this condition is automatically fulfilled because we observe only the perturbations of order costs described by (3) at this moment. For example, if the order costs on the first level can be reduced by $70 \%$ and the order costs on the second level by $10 \%$, then the total costs can be reduced by $31 \%$ (see Figure 5).

In all three cases we prove that on the one hand total average costs can be considerably reduced if we suppose the decrease of order costs, and on the other hand that our objective function is very sensitive to the variation of system parameters. Therefore it is shown that the reduction of order costs (for example due to the use of automated production or improved communication with all parties involved in the production) can indeed lead to reduced inventory-related costs. As our model helps us to estimate the approximate savings, it is also possible to make better and more informed decisions about whether a certain inventory related project should be accepted or not.

Now, let us calculate how much the total costs can be reduced if we change (reduce) the order costs for the first item by $10 \%\left(\frac{\Delta K_{1}}{K_{1}}=0,1\right.$ or $\left.\Delta K_{1}=2\right)$ and for the second item by $40 \%$ $\left(\frac{\Delta K_{2}}{K_{2}}=0.4\right.$ or $\left.\Delta K_{2}=6\right)$. We would also like to calculate the new optimal ordering intervals and compare their values with those we get if we don't consider optimization on parameter $m$.

Because the inequality $\sqrt{\frac{1-\frac{\Delta K_{2}}{K_{2}}}{1-\frac{\Delta K_{1}}{K_{1}}}}<\frac{\left\lfloor m_{0}^{*}\right\rfloor}{m_{0}^{*}}$ (after simplification: $\left.0,33+0,67 \frac{\Delta K_{1}}{K_{1}}=0,397<0,4=\frac{\Delta K_{2}}{K_{2}}\right)$ is fulfilled, we get: $\left|m_{p}^{*}\right|=1$. By equation (4) the optimal perturbed ordering interval on the first level becomes $\left(T_{p}^{*}\right)_{1}=0,42$, which means that it increases in comparison with $\left(T_{0}^{*}\right)_{1}=0,37$ by $14 \%$ (by equation (5)). The optimal perturbed ordering interval on the second level $\left(T_{p}^{*}\right)_{2}=0,42$ decreases in comparison with $\left(T_{0}^{*}\right)_{2}=2 \cdot 0,37=0,74$ by $43 \%$. If we calculate the optimal total perturbed costs by equation (6), we get $\left(C_{p}^{*}\right)_{1}=127,28$. This means that $\left(C_{p}^{*}\right)_{1}$ is reduced in comparison with $C_{0}^{*}=148,32$ by $14 \%$ (equation (7)). If we did not consider the optimization on $m$ - in which case we would use unperturbed $\left|m^{*}\right|=2$ in our expressions for optimal perturbed ordering intervals and total average perturbed costs, the optimal ordering intervals would decrease by $8 \%\left(\left(T_{p}^{*}\right)_{1}=0,34\right.$ and $\left.\left(T_{p}^{*}\right)_{2}=0,68\right)$, but the total costs would decrease in this case only by $9,5 \%\left(C_{p}^{*}=134,16\right)$.

\section{Conclusion}

From the presented derivations and figures it is possible to conclude that the behavior of the optimal total average costs heavily depends on the changes of order costs and on the optimal value of parameter $m\left(m^{*}\right)$. It is easy to see that if we chose the original (unperturbed) value $m_{0}^{*}$ $\left(m_{0}^{*}=2\right)$, instead of the real value for $m_{p}^{*}\left(m_{p}^{*}=1\right)$, a considerable relative error would take place with the change in the optimal value of average costs. On the one hand we have proven that 
total average costs can be fairly reduced if we suppose the decrease of order costs and optimization on parameter $m$, and on the other hand our objective function has proven to be very sensitive to the variation of system parameters. This shows that in this case complete and accurate information about order costs, demand, inventory related costs and other factors is vital. While it might be possible to obtain this information without the use of information technology and e-business, it is certainly much easier to achieve it with the proper use of modern technology. More complete information also means that the model presented in this paper can be used more broadly.

One should be therefore very careful while adopting the optimal ordering policy in a changeable environment (needeless to say, the enviroment today is becoming more and more changeable). In such a case it is obligatory to adopt the sensitivity study described in this paper in order to get a quick answer to the question of the influence of perturbed systems parameters to the optimal ordering policy and the optimal value of the objective function (the total average costs).

As e-business means easier access to information on the one hand, and frequent and drastical changes on the other, it is clear, that the model presented in this paper represents an invaluable tool for both researchers and praciticians in the fields of e-business and inventory management.

The results of this paper can be extended in two different directions. One possible direction for future research is to evaluate the model using it in a real scenario or make a simulation. Another potential direction for future research is to investigate the influence of Internet and e-business on the supply time with a view to minimizing the total inventory-related costs.

\section{References}

Bogataj, L., \& Ferbar, L. (1996). Stochastic considerations of Grubbstrom-Molinder model of MRP, inputoutput and multi-echelon inventory systems. International Journal of Production Economics, 45, 329336.

Emigh, J. (1999, August 23). Vendor managed inventory. Computerworld, Marion.

Ferbar, L., \& Bogataj, L. (1996). MRP, input-output analysis and multi-echelon inventory systems with exponentially distributed external demand. Proceedings of GLOCOSM Conference. Bangalore, India, 149-154.

Ferbar, L., \& Bogataj, L. (1997). Finite magnitude perturbations in the inventory systems. Proceedings of the 4th International Symposium on Operational Research in Slovenia, Preddvor. Ljubljana: Slovenian Society Informatika, Section for Operational Research, 45-52.

Ferbar, L., \& Bogataj, L. (1999). A market game with the characteristic function according to the MRP and input-output analysis model. International Journal of Production Economics, 59, 281-288.

Goutsos, S., \& Karacapilidis, N. (2003). Enhanced supply chain management for e-business transactions. International Journal of Production Economics. In press.

Grubbstrom, R.W., \& Molinder, A. (1994) Further theoretical considerations on the relationship between MRP, input-output analysis and multi-echelon inventory systems. International Journal of Production Economics, 35, 299-311.

Keen, P. (2000, February 14). IT's value in the chain. Computerworld, Marion.

Krauter, J. (1999). Inventory theory: New perspectives for corporate management. International Journal of Production Economics, 59, 129-134.

McCubbrey, D. (1998). Elektronsko poslovanje - kaj je staro in kaj je novega. Organizacija, Kranj, 31 (3), $172-175$.

Panayiotou, N, Gayialis, S., \& Tatsiopoulos I. (2003). An e-procurement system for governmental purchasing. International Journal of Production Economics. In press. 
Ramani, K., Yap, R., \& Pavri F. (1995). Case study. Information technology enables business process reengineeting at YCH DistriPark (Singapore). Journal of Strategic Information Systems, 4, 81-88.

Sianesi, A., \& Zavanella, L. (1996). MRP: a simulative investigation on sensitivity to system parameters. Ninth International Symposium on Inventories, ISIR, Budapest.

Trkman, P. (2000). Uspešnost poslovanja in informatizacija : diplomsko delo. Ljubljana.

Trkman, P., Turk, T., Vehovar, V., \& Jerman-Blazic, B. (2001). Uspešnost poslovanja in informatizacija. Proceedings. Dnevi slovenske informatike, Portorož, Slovenija. Ljubljana: Slovensko društvo Informatika: $=$ Slovenian Society Informatika, 276 .

Trombly, M. (2000, May 22). Value-chain management. Computerworld, Marion.

Van der Vorst, J. G. A. J, Van Dongen, S., Nouguier, S., \& Hilhorst, R. (2002). E-business initiatives in food supply chains; definition and typology of electronic business models. InternationalJournal of Logistic: Research and Applications, 5 (2), 119-138.

Zupancic, B. (1997). Elektronsko poslovanje v oskrbovalnih verigah. Organizacija, Kranj, 30 (5), 254-263.

\section{Biography}

Liljana Ferbar, Ph. D., is associate professor for Mathematics at Faculty of Economics, University of Ljubljana, Slovenia. She teaches courses in Linear Algebra, Mathematics for Business and Material Requirement Planning. Her research interests lie in Linear Algebra, Operation Research and Information Technology in Mathematics Education. 